\title{
Congenital Inferior Vena Cava Agenesis with Ulceration and Deep Vein Thrombosis
}

\author{
Melanie Menning ${ }^{1}$, Mira Yousef ${ }^{2}$ \\ ${ }^{1}$ Department of Family Medicine, University of Nebraska Medical Center, Omaha, Nebraska, USA \\ ${ }^{2}$ College of Medicine, University of Nebraska Medical Center, Omaha, Nebraska, USA
}

Received: 20/01/2021

Accepted: 26/01/2021

Published: $18 / 03 / 2021$

How to cite this article: Menning M, Yousef M. Congenital inferior vena cava agenesis with ulceration and deep vein thrombosis. EJCRIM 2021;8: doi:10.12890/2021_002312.

Conflicts of Interests: The Authors declare that there are no competing interests.

This article is licensed under a Commons Attribution Non-Commercial 4.0 License

\section{ABSTRACT}

Objectives: This is one of the few case reports of congenital inferior vena cava atresia (IVCA) with extensive bilateral lower extremity ulcers. Background: IVCA is a congenital anomaly characterized by poor venous return in the lower extremities with increased risk of deep vein thrombi (DVTs) and, rarely, non-healing ulcers.

Results: A 45-year-old woman with history significant for DVTs presented with extensive bilateral lower extremity ulcers, and a CT scan revealed congenital IVCA. Oedema management, DVT prevention, routine wound care and skin grafts resolved the ulcers.

Conclusion: Congenital venous abnormalities should be included in the differential diagnosis of multiple unprovoked DVTs and non-healing ulcers.

\section{LEARNING POINTS}

- There are very few reports in the English literature describing inferior vena cava atresia with extensive bilateral lower extremity ulcers.

- We present our findings as well as new insights into the congenital causes of bilateral lower extremity ulcers and deep vein thrombi (DVTs).

- Especially for young adults with a history of multiple DVTs and non-healing ulcers, venous abnormalities should be included in the differential diagnosis and advanced imaging should be utilized.

\section{KEYWORDS}

Inferior vena cava atresia, IVCA, ulcers, deep vein thrombus, DVT

\section{INTRODUCTION}

Inferior vena cava atresia (IVCA) is a very rare anomaly, affecting the venous return of the lower extremities and predisposing patients to ulceration and deep vein thrombi (DVTs). The severity depends on whether it is a partial or complete absence but altogether, the prevalence is $0.0005-1 \%$ in the general population ${ }^{[1]}$. Specifically, it is found in up to $5 \%$ of patients under the age of 30 with a history of unprovoked DVT ${ }^{[1]}$. Since DVTs are often confirmed by ultrasound, the diagnosis of IVCA, which requires either a computed tomography (CT) scan or magnetic resonance imaging (MRI), is often incidental and can easily be missed. The pathogenesis of IVCA involves significantly impaired venous return and can have a varied presentation ${ }^{[2]}$. With close to $70 \%$ of vascular malformations being of venous origin, findings such as large superficial compensatory veins are often indicative of the underlying pathology. Due to the development of collaterals, these malformations can remain asymptomatic for a long period of time. If detected late or managed insufficiently, the impaired blood flow confers 
a 10-fold increase in the risk of a DVT which can later present with extensive, non-healing ulceration ${ }^{[3,4]}$.

Here we describe a unique presentation of non-healing venous ulcers due to symptomatic IVCA. Clinical management includes compression stockings, lifelong anticoagulation and avoidance of known risk factors for DVT. Unfortunately, there are no current surgical interventions. This report describes an atypical case of IVCA in a 45-year-old woman with a history of three DVTs who presented years later with extensive, circumferential bilateral lower extremity venous ulcers. A CT scan ultimately revealed her congenital abnormality, effectively diagnosing her symptoms after nearly 15 years of complications.

\section{CASE PRESENTATION}

A 45-year-old woman presented with painful, circumferential bilateral lower extremity ulcers that made it nearly impossible to walk (Fig. 1) Her past medical history was notable for three DVTs within 10 years, the first one occurring postpartum. Her associated symptoms from the venous stasis were extreme pain from the ulcers and swelling. Her previous treatments included phlebectomy, bilateral ablation of her saphenous veins and skin grafting, but her non-healing wounds continued to worsen. When she initially presented, her wounds had circumferential ulceration around both ankles with yellow slough and erythematous tissue. The dimensions on the right ankle were $5 \mathrm{~cm}$ on the lateral aspect, $14.5 \mathrm{~cm}$ on the anterior aspect, and $6.5 \mathrm{~cm}$ on the medial aspect in proximal distal measurement. The left ankle ulcer had similar dimensions, being $18.5 \mathrm{~cm}$ at the largest proximal distal measurement. Wounds were painful with even light touch and when removing gauze. The patient was additionally found to be diabetic with a glycated haemoglobin (HbA1c) of 9.8\%.

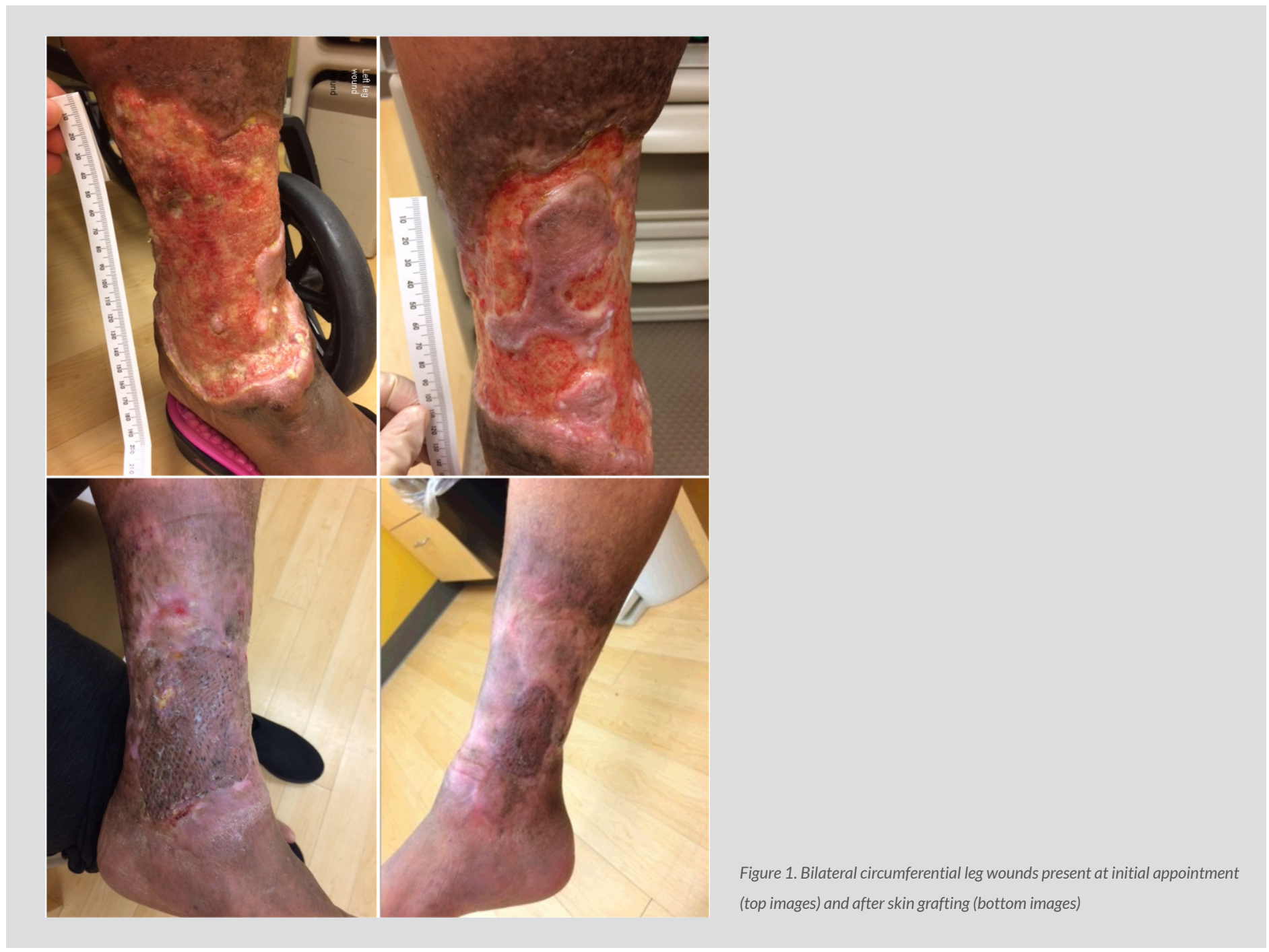


Clinical Evaluation and Management

Venous duplex ultrasound showed no evidence of acute DVT. A CT scan of the abdomen and pelvis was obtained, revealing near absence of the IVC below the renal veins (Fig. 2). The CT scan also demonstrated the formation of collaterals with an enlarged azygous vein and pelvic/ iliac collaterals (Fig. 3). Wounds were managed with usual wound care, diabetic control and compression with dramatic improvement. The patient was additionally started on long-term anticoagulation (rivaroxaban). Ultimately, complete closure of wounds was achieved with skin grafting (Fig. 1). The patient has not had any further thrombotic events or ulceration and her wounds have completely resolved. Interestingly, her diabetes also resolved with wound closure: she has maintained a $\mathrm{HbA} 1 \mathrm{c}$ below $6.0 \%$ and is off all diabetes medications since closure for over a year.
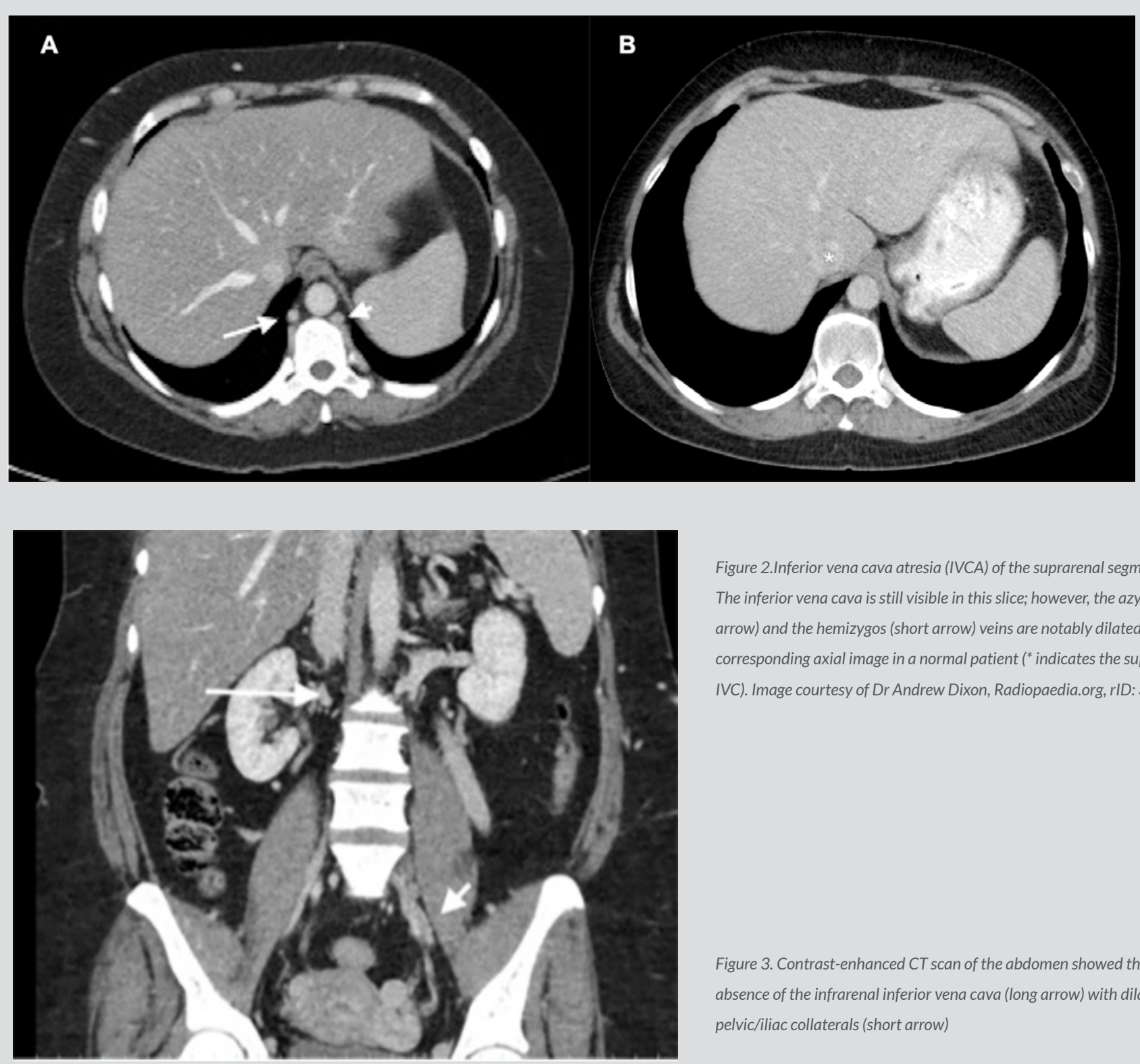

Figure 2.Inferior vena cava atresia (IVCA) of the suprarenal segment. (A) The inferior vena cava is still visible in this slice; however, the azygos (long arrow) and the hemizygos (short arrow) veins are notably dilated. (B) The corresponding axial image in a normal patient $\left({ }^{*}\right.$ indicates the suprarenal IVC). Image courtesy of Dr Andrew Dixon, Radiopaedia.org, rID: 36677

Figure 3. Contrast-enhanced CT scan of the abdomen showed the absence of the infrarenal inferior vena cava (long arrow) with dilated pelvic/iliac collaterals (short arrow)

\section{DISCUSSION}

This case report describes a case of IVCA that presented with chronic, non-healing bilateral leg ulcers and a history of multiple DVTs. IVCAassociated DVT has been reported periodically in the literature, with 62 patients as of $2010^{[1]}$. However, IVCA with a non-healing ulcer presentation is still quite rare, with only a handful of reported cases. 
The aetiology of IVCA is still debated and there are several hypotheses. One hypothesis describes the atresia as a consequence of intrauterine or perinatal thrombosis ${ }^{[5]}$; however, this does not explain the absence of deep veins that may accompany IVCA ${ }^{[5]}$. More extensively supported is the dysembryogenesis hypothesis, which reasons that abnormal development during the 6th through 8th weeks of gestation leads to the many vascular malformations reported in the literature ${ }^{[6]}$. Formation of the IVC requires a series of complex embryological changes in veins including the vitelline, posterior-cardinal, subcardinal and supracardinal veins ${ }^{[7,8]}$. A particularly well-known developmental challenge is the formation of the infrarenal segment of the IVC from the right supracardinal vein ${ }^{[7,8]}$.

Young adults with this anomaly often remain asymptomatic until later in life or until another trigger arises such as pregnancy, intense exercise or immobilization ${ }^{[9]}$. The most feared complication is the 10 -fold increase in the risk of a DVT, which warrants immediate attention. The final diagnosis of IVCA is made with subsequent ultrasound, CT or MRI imaging ${ }^{[3]}$. Other well-reported consequences include skin induration, collateral vein circulation, and most strikingly, non-healing ulcers ${ }^{[4]}$. Clinical management of IVCA is mostly supportive and preventive with lifelong anticoagulation, minimization of thrombotic risk factors, compression stockings and wound management. There are no other surgical interventions for IVCA. The presence of extensive venous ulceration, especially in the context of unprovoked DVTs, warrants work-up for a diagnosis of IVCA with CT or MRI of the abdomen and pelvis ${ }^{[10]}$.

\section{CONCLUSION}

This case report highlights the risk of congenital IVCA with non-healing ulcers and recurrent DVTs. Clinicians should be aware of IVCA and keep it in mind as a potential clinical diagnosis when presented with a young patient with unexplained DVTs and impaired blood flow leading to extensive lower extremity venous ulceration. Even though there is no specific intervention for IVCA, it can be managed with supportive care, compression and lifelong anticoagulation.

\section{REFERENCES}

1. Lambert M, Marboeuf P, Midulla M, Trillot N, Beregi JP, Mounier-Vehier C, et al. Inferior vena cava agenesis and deep vein thrombosis: 10 patients and review of the literature. Vasc Med 2010;15(6):451-459.

2. Eifert S, Villavicencio JL, Kao T-C, Taute BM, Rich NM. Prevalence of deep venous anomalies in congenital vascular malformations of venous predominance. J Vasc Surg 2000;31(3):462-471.

3. Bianchi M, Giannini D, Balbarini A, Castiglioni MG. Congenital hypoplasia of the inferior vena cava and inherited thrombophilia: rare associated risk factors for idiopathic deep vein thrombosis. A case report. J Cardiovasc Med 2008;9(1):101-104.

4. Tufano A, López-Jiménez L, Bikdeli B, García-Bragado F, Mazzolai L, Amitrano M, et al. Inferior vena cava agenesis in patients with lower limb deep vein thrombosis in the RIETE registry. When and why to suspect. Int J Cardiol 2020;305:115-119.

5. Gupta A, Kumar S, Kothari SS. Congenital absence of infrarenal inferior vena cava and deep veins of the lower limbs: a case report. J Med Case Rep 2016;10(1):1-4.

6. Spentzouris G, Zandian A, Cesmebasi A, Kinsella CR, Muhleman M, Mirzayan N, et al. The clinical anatomy of the inferior vena cava: a review of common congenital anomalies and considerations for clinicians. Clin Anat 2014;27(8):1234-1243.

. Sneed D, Hamdallah I, Sardi A. Absence of the retrohepatic inferior vena cava: what the surgeon should know. Am Surg 2005;71(6):502-504.

8. Moore KL, Persaud TVN. The cardiovascular system. In: The developing human: clinically oriented embryology. 6th ed. Philadelphia: WB Saunders; 1998, p. 351-358.

9. Muscianese L, Seese RR, Graham W, Williams JH. Congenital atresia of the inferior vena cava and antithrombin III deficiency in a young adult: compounding risk factors for deep vein thrombosis. BMJ Case Rep 2015;2015:bcr2014205729.

10. Raju S, Hollis K, Neglen P. Obstructive lesions of the inferior vena cava: clinical features and endovenous treatment. J Vasc Surg 2006;44(4):820-827. 